Pacific Journal of Mathematic 


\title{
AN APPLICATION OF GROUPOID COHOMOLOGY
}

\section{Caroline Series}

\begin{abstract}
We study the structure of analytic measured groupoids as defined by Mackey. It has been observed by Ramsay that an arbitrary groupoid can be thought of as an equivalence relation on its unit space together with a field of isotropy subgroups.

We construct a cohomology theory for equivalence relations with coefficients in a field of abelian groups, and show that two possible definitions using strict cochains or almost everywhere cochains coincide, and show how using this to reconstruct a groupoid from an equivalence relation and a field of groups,
\end{abstract}

Introduction. We study the structure of analytic measured groupoids as defined by Mackey [4]. It has been observed by Ramsay [8], Theorem 6.9 that an arbitrary groupoid can be thought of as an equivalence relation on its unit space together with a field of isotropy subgroups. A groupoid homomorphism consists of an orbit preserving mapping of the unit spaces together with a homomorphism of the fields of isotropy subgroups. We formalize this correspondence in the language of group extensions. The discussion is motivated by the observation that if $\mathscr{G}$ is any groupoid we can associate to it $R \mathscr{G}$, the corresponding equivalence relation, and $\Gamma \mathscr{G}$, the field of isotropy subgroups, and there are natural maps $\Gamma \mathscr{G} \rightarrow \mathscr{G} \rightarrow R \mathscr{G}$. This is a short exact sequence of groupoids, in a sense explained in $\S 1$, so that $\mathscr{G}$ may be thought of as an extension of the field $\Gamma \mathscr{G}$ by the equivalence relation $R \mathscr{G}$.

In $\S 2$ we construct a cohomology theory for equivalence relations with coefficients in a field of abelian groups, and show that two possible definitions using strict cochains or almost everywhere cochains coincide. In $\S 3$ we consider how to reconstruct a groupoid from an equivalence relation and a field of groups. More precisely, an abstract kernel will consist of an equivalence relation and a field of groups together with suitable connecting isomorphisms. Any groupoid gives rise to an abstract kernel and conversely any abstract kernel gives rise to a groupoid provided that a certain obstruction in $H^{3}$ vanishes. The methods we use are algebraically an exact analogue of the usual theory of group extensions [5]. It is the author's hope that the language of abstract kernels may prove a more useful viewpoint for the study of groupoids.

Cohomology for groupoids with coefficients in a single abelian group has been discussed by Westman [12], and for the special case 
of an equivalence relation with countable orbits by Feldman and Moore [2]. The methods we use in $\S 2$ follow Moore [6] very closely. L. Brown obtained the equivalence of the two cohomology theories for an equivalence relation generated by a freely acting group with coefficients in a single abelian group. Presumably our results extend to a cohomology theory for an arbitrary groupoid with coefficients in a field of abelian groups.

If $X$ and $Y$ are standard Borel spaces, $\mathscr{F}(X, Y)$ is the space of Borel functions $f: X \rightarrow Y$, and $\mathscr{F}_{1 \prime}(X, Y)$ is the space of functions measurable with respect to some measure $\mu$ on $X$, with identification of functions agreeing a.e. on $X$. If $\nu$ is a measure on $Y$ and $Z$ is another Borel space, and $f \in \mathscr{F}(X, Z), g \in \mathscr{F}(Y, Z)$, then $X_{f, g}^{*} Y$ is the fiber product $\{(x, y) \in X \times Y: f(x)=g(y)\}$ and $\mu * \nu$ is the fiber product measure (c.f. [7] p. 265).

1. Preliminaries. We will assume the reader is familiar with the theory of ergodic groupoids, as expounded in [4] and [7]. To fix our notation, we recall some definitions.

Let $\mathscr{C}, h$ be a measured analy tic groupoid (not necessarily ergodic). The unit space of $\mathscr{C}$ will be written $S_{\mathscr{C}}$ or simply $S$. The right and left projections to the units will be written $d, g$ respectively (for droite and gauche: this is an attempt to reconcile the notation of Ramsay who uses $d, r$ for domain and range and the author's prejudices in favor of right and left). We write $\bar{\xi}=d(\xi), \xi=g(\xi) ; \mathscr{G}^{(2)}=\mathscr{G} *_{d, g} \mathscr{O}$, and more generally $\mathscr{G}^{(n)}=\mathscr{C}_{d_{, g^{(n-1)}}} \mathscr{G}^{(n-1)}$ where $g^{(n-1)}: \mathscr{S}^{(n-1)} \rightarrow S$ is $g\left(\xi_{1}, \cdots, \xi_{n}\right)=\xi_{1}$ and $d^{(n-1)}$ is defined similarly. $h^{(n)}$ is the fiber product measure $h * h^{(n-1)}$, and $\bar{h}=d * h . \quad F^{s}=g^{-1}(s), F_{s}=d^{-1}(s)$, with measures $h^{s}, h_{s}$ respectively. $\quad T^{\hat{\xi}}: F^{\bar{\xi}} \rightarrow F^{\hat{\xi}}, T^{\xi}(\eta)=\xi \eta$. $\mathscr{C}$ acts on a space $X, \nu$ if there is a Borel map $P: X \rightarrow S$ and a Borel map $m: X *_{p, g} \mathscr{S} \rightarrow X$, such that

$$
m(x, s)=x \quad \text { and } \quad m(m(x, \xi), \eta)=m(x, \xi \eta),
$$

and such that $P_{*} \nu=\bar{h}$ and if $\nu=\int_{S} \nu_{s} d \bar{h}(s)$ and $J_{\xi}: P^{-1}(\xi) \rightarrow P^{-1}(\bar{\xi})$, $J_{\xi}(x)=x \xi$, then $J_{\xi} * \nu_{\xi} \sim \nu \bar{\xi} \forall \xi$ in an i.c. of $\mathscr{G}$. (c.f. [9] Definition 1.2).

Ramsay [9] has discussed the kernel and range closure of a groupoid homomorphism $\pi: \mathscr{G} \rightarrow \mathscr{C}$. For our purposes it suffices to consider only the case $S_{\mathscr{G}}=S_{\mathscr{C}}, \bar{\pi}=\mathrm{id}$, in which case the construction simplifies considerably. Ker $\pi$ is the $\mathscr{C}$ space $\mathscr{H}$ with $P: \mathscr{C} \rightarrow S$, $P(\xi)=\xi, m(\xi, \eta)=\pi(\eta)^{-1} \xi . \quad \mathscr{C}$ is also an $\mathscr{C}$ space with $d: \mathscr{H} \rightarrow S_{\mathscr{C}}$ and $m(\xi, \eta)=\xi \eta$. By [9] Theorem 3.2 there is an $\mathscr{H}$ space $\mathscr{\mathscr { C }}$ and an equivariant map $j(\pi): \mathscr{H} \rightarrow \overline{\mathscr{C}}$ such that each fiber of $j(\pi)$ is an ergodic groupoid. The action of $\mathscr{H}$ on $\overline{\mathscr{C}}$ is called the range closure 
of $\pi$, written $\mathscr{R}(\pi) . \quad \pi$ is an embedding if each fiber of $j(\pi)$ is the trivial groupoid, i.e., if it is principal and transitive. $\pi$ is surjective if each fiber of $j(\pi)$ is transitive and we may take $\overline{\mathscr{H}}=S$, with the natural $\mathscr{H}$ action, and $j(\pi)=d$. A sequence $\mathscr{\mathscr { C }} \stackrel{i}{\rightarrow} \mathscr{G} \stackrel{j}{\rightarrow} \mathscr{H}$ of groupoids is a short exact sequence if $i$ is an embedding, $j$ is surjective, and $\operatorname{Ker} j=\mathscr{R}(i)$.

Associated to any groupoid $\mathscr{G}$ are two other groupoids $R \mathscr{G}, \Gamma \mathscr{G}$. $R \mathscr{G}=\{(s, t) \in S \times S: \exists \xi \in \mathscr{G}, \bar{\xi}=t, \xi=s\}$ is the associated principal groupoid (equivalence relation). This is shown to be a groupoid in [7]. $\Gamma \mathscr{G}=\bigcup_{s \in S} \Gamma \mathscr{G}_{s}$, where $\Gamma \mathscr{G}_{s}=\{\xi \in \mathscr{G}: \bar{\xi}=\xi=s\}$. By [7], each $\Gamma \mathscr{G}_{s}$ is a l.c.s.c. group and $\Gamma \mathscr{G}$ is a measured field of groups in the sense of the definition of $\S 2$. $\Gamma \mathscr{G}$ inherits its algebraic structure from $\mathscr{G}$; we remark that it is not ergodic unless $\mathscr{G}$ is a group.

THEOREM 1.1. There is a natural short exact sequence $\Gamma \mathscr{G} \stackrel{i}{\rightarrow}$ $\mathscr{C} \stackrel{R}{\rightarrow} R \mathscr{G}$.

Proof. The definitions of $i$ and $R$ are clear. Ker $i$ is the $\Gamma \mathscr{G}$ space $\mathscr{G}$, with $\xi \cdot \eta=\eta^{-1} \xi, \eta \in \Gamma \mathscr{G}, \xi \in \mathscr{G}$. We may take $\overline{\mathscr{G}}=R \mathscr{G}$, with $j(i)=R . \quad R \mathscr{G}$ is in a natural manner a $\mathscr{G}$ space, with $\mathscr{G}$ acting on the left, and each fiber of $j(i)$ is the trivial groupoid. $\operatorname{Ker} R$ is the $\mathscr{G}$ space $R \mathscr{G}$, with the natural left action of $\mathscr{G}$, and hence $\operatorname{Ker} R=\mathscr{R}(i) . \quad R \mathscr{G} \rightarrow S, u \mapsto \bar{u}$, is the ergodic decomposition of $\operatorname{Ker} R$ and is an $R$ equivariant map with the natural action of $R \mathscr{G}$ on $S$. Each fiber is transitive, hence $R$ is surjective.

2. Cohomology of equivalence relations. We extend the cohomology theories of [3] and [12] by showing how to define cohomology groups for equivalence relations taking values in a field of abelian groups. The main result, obtained following methods of Moore, [6], is that two possible definitions of the cohomology groups, using strict Borel cochains or cochains of functions identified almost everywhere, coincide.

Throughout $R, h$ is a principal groupoid, with unit space $S$; $\mu$ is the induced measure measure on $S$, and $h^{(n)}$ the induced measure on $R^{(n)}=\left\{\left(v_{1}, \cdots, v_{n}\right) \in R^{n}: \bar{v}_{i}=v_{i+1}, i=1, \cdots, n-1\right\}$.

A Polish field of groups on $S$ is an assignment $s \mapsto A_{s}$ of a Polish group (c.f. [6] p. 3) to each $s \in S$, such that

(i) $\underline{A}=\bigcup_{s \in S} A_{s}$ has a standard Borel structure $\mathscr{B}$.

(ii) $\left.\mathscr{B}\right|_{A_{s}}$ is the natural Borel structure on $A_{s}$.

(iii) $\alpha: \underline{A} \rightarrow S, \alpha\left(A_{s}\right)=s$, is Borel.

A measured field of l.c.s.c. groups is a Polish field of groups such that $A_{s}$ is l.c.s.c for each $s$, and such that there is a choice of $\nu_{s}$ in 
the Haar class on $A_{s}$ with $s \mapsto \nu_{s}(E)$ Borel for each $E \in \mathscr{B}(\underline{A})$.

Such a field is an $R$-module if we are given, for each $v \in R$, a topological isomorphism $J(v): A_{\bar{v}} \rightarrow A_{\underline{v}}$ such that $J\left(v_{1}\right) J\left(v_{2}\right)=J\left(v_{1} v_{2}\right) \forall v_{1}$, $v_{2} \in R^{(2)}$ and such that the map

$$
R *_{d, \alpha} \underline{A} \longrightarrow \underline{A}, \quad(v, a) \longmapsto J(v) a, \quad \text { is Borel . }
$$

If $\underline{B}, K$ is an $R$-module, an $R$-module map $T: \underline{A} \rightarrow \underline{B}$ consists of continuous homomorphisms $T(s): A_{s} \rightarrow B_{s}$ such that $K(v) T(v)=$ $T(v) J(v) \forall v \in R$ and such that

$$
\underline{T}: \underline{A} \longrightarrow \underline{B}, \quad \underline{T}(a)=T(\alpha(\alpha))(\alpha), \quad \text { is Borel . }
$$

Given an $R$-module $\underline{A}$, we can construct another $R$-module $I(\underline{A})$, analogous to the regular representation of a group.

$$
I(\underline{A})_{s}=\left\{f \in \mathscr{F}_{h_{s}}\left(d^{-1}(s), \underline{A}\right): f(v) \in A_{\underline{v}}\right\}
$$

$I(A)_{s}$ may be identified with $\mathscr{F}_{h_{s}}\left(d^{-1}(s), A_{s}\right)$ by the map

$$
f \longmapsto \bar{f}, \quad \bar{f}(v)=J(v)^{-1} f(v) .
$$

$I(\underline{A})_{s}$ is topologised as follows (c.f. [6] p. 5):

$$
f_{n} \rightarrow f \Longleftrightarrow J(v)^{-1} f_{n}(v) \longrightarrow J(v)^{-1} f(v) \quad h_{s} \text { a.e. }
$$

By [6] p. 6, this makes $I(\underline{A})_{s}$ a Polish group. Since $J(v)$ is a topological isomorphism, 2.1 is equivalent to

$$
f_{n} \longrightarrow f \Longleftrightarrow f_{n}(v) \longrightarrow f(v) \quad h_{s} \text { a.e. }
$$

We define the Borel structure on $I(\underline{A})=\bigcup_{s \in S} I(\underline{A})_{s}$ to be the smallest such that the projection $i(\alpha): I(\underline{A}) \rightarrow S$ and all functions $I(\underline{A}) \rightarrow \boldsymbol{R}$ of the form

$$
G(Y, \dot{\phi})(h)=\int_{Y} \dot{\phi}(h(v)) d h_{\bar{v}}(v), \quad Y \in \mathscr{B}(R), \quad \phi \in \mathscr{F}(\underline{A}, \boldsymbol{R}),
$$

are Borel. This makes $I(\underline{A})$ into a standard Borel space and by [6] Proposition 8 the given Borel structure is induced on $I(\underline{A})_{s}$ for each $s$. Notice that all functions $h \mapsto \int_{Y} \rho(v) \dot{\phi}(h(v)) d h_{\bar{v}}(v), \rho \in \mathscr{F}(\underline{A}, \boldsymbol{R})$, are automatically Borel.

Proposition 2.1. $I(\underline{A})$ is an $R$-module.

Proof. $\quad R$ acts on $I(\underline{A})$ by

$$
T(v): I(\underline{A})_{\bar{v}} \longrightarrow I(\underline{A})_{\underline{v}}, \quad[T(v) f](w)=f(w v) .
$$

Using 2.2 and the quasi-invariance of the measures $h_{s}$ it is clear that 
$T(v)$ is a topological isomorphism. $R * I(\underline{A}) \rightarrow I(\underline{A})$ is Borel provided the maps

(i ) $v, h \mapsto \alpha(T(v) h)$

(ii) $v, h \mapsto G(Y, \phi)(T(v) h), Y \in \mathscr{\mathscr { B }}(R), \phi \in \mathscr{F}(\underline{A}, \boldsymbol{R})$, are Borel.

is clear and (ii) follows since

$$
\begin{aligned}
G(Y, \phi)(T(v)(h)) & =\int_{Y} \phi((T(v) h)(w)) d h_{\bar{w}}(w)=\int_{Y} \phi(h(w v)) d h_{\bar{w}}(w) \\
& =\int_{Y} \phi(h(w)) \rho_{v}(w) d h_{\bar{v}}(w)
\end{aligned}
$$

where $\rho_{v}$ is the Radon-Nikodym derivative of $T_{v^{*}}\left(h_{v}\right)$ with respect to $h_{\bar{\imath}}^{-}$.

Proposition 2.2. There is a natural embedding of $R$-modules $\underline{A} \rightarrow I(\underline{A})$.

Proof. Define $I(s): A_{s} \rightarrow I(\underline{A})_{s}, I(s)(a)(v)=J(v) a$. It is routine to check that $I(s)$ is algebraically an $R$-module map. That $I(s)$ is a homeomorphism onto its image follows as in [6] Proposition 13.

To see that $\underline{I}$ is Borel, one checks that $\underline{A} \mapsto \boldsymbol{R}, a \mapsto \int_{Y} \phi(J(v) a) d h_{\underline{v}}(v)$ is Borel for $Y \in \mathscr{B}(R), \phi \in \mathscr{F}(\underline{A}, \boldsymbol{R})$. This follows since $(v, a) \mapsto J(v) a$ is Borel.

We define another field $U(\underline{A}), U(\underline{A})_{s}=I(\underline{A})_{s} / A_{s}$. It is clear that with the induced Borel structure and induced $R$-action, $U(\underline{A})$ is an $R$-module.

We now turn to the construction of the cohomology groups. Following [6] and [2] we axiomatise a cohomology functor as follows:

Let $R$ be a principal groupoid. A cohomological functor on the category of $R$-modules (in the sense discussed above) is a sequence of contravariant functors $H^{n}(R, \underline{A})$, for each $n \geqq 0$, to the category of abelian groups, such that to each short exact sequence of $R$ modules there corresponds a long exact sequence of cohomology in the usual way, and such that

$$
H^{0}(R, \underline{A})=\underline{A}^{R} ; H^{n}(R, I(\underline{A}))=0
$$

where

$$
\underline{A}^{R}=\left\{f \in \mathscr{F}_{\mu}(S, \underline{A}): f(s) \in A_{s}, \quad J(v) f(\bar{v})=f(\underline{v}) \quad \text { a.a. } v \in R\right\} .
$$

As in [6] one verifies the uniqueness of a cohomological functor satisfying these axioms.

Just as in [6] we have two possible candidates for the cochain groups: 


$$
\begin{aligned}
C^{n}(R, \underline{A})= & \left\{c \in \mathscr{F}\left(R^{(n)}, \underline{A}\right): c\left(u_{1}, \cdots, u_{n}\right) \in A_{\underline{u}_{1}}\right\} \\
\partial C\left(u_{1}, \cdots, u_{n+1}\right)= & J\left(u_{1}\right) c\left(u_{2}, \cdots, u_{n+1}\right) \\
& +\sum_{i=1}^{n}(-1)^{i} c\left(u_{1}, \cdots, u_{i} u_{i+1}, \cdots u_{n+1}\right) \\
& +(-1)^{n+1} c\left(u_{1}, \cdots, u_{n}\right),
\end{aligned}
$$

where we identify functions which agree on saturated conull sets.

$$
\underline{C}^{n}(R, \underline{A})=\left\{c \in \mathscr{F}_{h^{(n)}}\left(R^{(n)}, \underline{A}\right): c\left(u_{1}, \cdots, u_{n}\right) \in A_{\underline{u}_{1}}\right\}
$$

and $\underline{\partial}$ is given by the same formula as $\partial$. One verifies that $\underline{\partial}$ is well-defined on $\underline{C}^{n}$.

It is clear that there is a natural map $H^{n} \rightarrow \underline{H}^{n}$, induced by identifying functions which agree a.e. It is also clear that from a short exact sequence of $R$-modules one obtains a long exact sequence of cohomology.

Our aim is to show that the two definitions of cohomology groups satisfy the axioms and that $H^{*} \rightarrow \underline{H}^{*}$ is an isomorphism.

Proposition 2.3. $H^{0}(R, \underline{A})=\underline{H}^{0}(R, \underline{A})=\underline{A}^{R}$.

Proof. Suppose $f \in \underline{Z}^{0}$. Then $\partial f=0$ is precisely the condition $f \in \underline{A}^{R}$.

Let $f \in Z^{0}(R, \underline{A})$. Then $J(v) f(\bar{v})=f(\underline{v})$ on a saturated conull set in $R$. Therefore $f$ gives a well-defined element $f \in \underline{A}^{R}$. Suppose $\underline{f}=\underline{f}^{\prime}$ in $\underline{A}^{R}$. Then $f(s)=f^{\prime}(s)$ a.a.s, and $J(v) f(\bar{v})=f(\underline{v}), J(v) f^{\prime}(\bar{v})=$ $f^{\prime}(\underline{v})$, on a saturated conull set in $R$. Consider $\left\{s \in S: f(s) \neq f^{\prime}(s)\right\}$. This is null and saturated under $R$, hence $f=f^{\prime}$ in $Z^{0}$. Conversely, suppose $f$ is a representative for $f \in A^{R}$. Then $J(v) f(\bar{v})=f(\underline{v})$ a.a.v. We need a function in $Z^{0}$ agreeing with $f$ a.e.

Define, for $s \in S, F(s) \in \mathscr{F}\left(d^{-1}(s), \underline{A}\right), F(s)(v)=J(v)^{-1} f(\underline{v})$. For $\mu$ a.a. $s, F(s)$ is constant $h_{s}$ a.e. Let this constant value be $a(s)$, so that $F(s)(v)=a(s)$ a.a. $s \in S$. Let $T=\left\{s \in S: F(s)\right.$ is constant $h_{s}$ a.e. $\}$. Suppose $w \in R, \bar{w} \in T$. Then

$$
F(\underline{w})(x)=J(x)^{-1} f(\underline{x})=J(w) J(x w)^{-1} f(\underline{x w})=J(w) F(\bar{w})(x w)
$$

which is constant $h_{\underline{w}}$ a.e. Therefore $\underline{w} \in T, T$ is saturated, moreover $a(\underline{w})=J(w) a(\bar{w})$ for $\bar{w} \in T$. It is clear that $a(s)=f(s)$ a.a.s, and $a$ is Borel since

$$
\phi(a(s))=\int \phi\left(J(v)^{-1} f(\underline{v})\right) d h_{\bar{v}}(v) \quad \text { is Borel } \quad \forall \phi \in \mathscr{F}(\underline{A}, \boldsymbol{R})
$$

$a$ is therefore the required function.

The next step is a modified version of [6] Theorem 1: 
Proposition 2.4 (Modified Fubini Theorem).

$$
\underline{C}^{n}(R, \underline{A})=\underline{C}^{n-1}(R, I(\underline{A})), \quad C^{n}(R, \underline{A})=C^{n-1}(R, I(\underline{A})) .
$$

Proof. We have an obvious map $L: C^{n} \rightarrow C^{n-1}, L(f)\left(v_{2}, \cdots, v_{n}\right)=$ $f\left(v_{1}, \cdots, v_{n}\right)$ and similarly for $\underline{C}^{n}$. One checks that $L, \underline{L}$ are well-defined. It is clear that $L$ is an isomorphism. It is also straightforward to check $\underline{L}$ is injective and Borel. To see that $\underline{L}$ is surjective we follow [6] p. 8. $\underline{A}$ is Polish and may therefore be embedded in an infinite cube $I^{\infty}$. Let $p_{i}$ denote projection to the $i$ th coordinate. For $M \in \mathscr{B}(R)$, $N \in \mathscr{B}\left(R^{(n-1)}\right), f \in \underline{C}^{n-1}(R, I(\underline{A}))$, set

$$
\begin{aligned}
\nu_{i}(M, N) & =\int_{M} \int_{N} p_{i} f\left(v_{2}, \cdots, v_{n}\right)\left(v_{1}\right) d h_{\bar{v}_{1}}\left(v_{1}\right) d h^{(n-1)}\left(v_{2}, \cdots, v_{n}\right) \\
& =\int_{M \times N} p_{i} f\left(v_{2}, \cdots, v_{n}\right)\left(v_{1}\right) d h^{(n)}\left(v_{1}, \cdots, v_{n}\right) .
\end{aligned}
$$

The inner integral is Borel by definition of the Borel structure on $\underline{C}^{n-1}(R, I(\underline{A})) . \quad \nu_{i}(M, N)$ extends to a measure $\nu_{i}$ on $R^{(n)}$ which is absolutely continuous with respect to $h^{(n)}$. Let $\rho_{i}$ be the RadonNikodym derivative of $\nu_{i}$ with respect to $h^{(n)}$. Define $\rho: R^{(n)} \rightarrow A$, $\rho=\left(\rho_{1}, \rho_{2}, \cdots\right)$. It is straightforward to check that $\underline{L}(\rho)=f$.

Just as we formed $I(\underline{A})_{s}$ and gave $I(\underline{A})$ the structure of a Polish field of groups, we may form

$$
I^{(2)}(\underline{A})_{s}=\left\{f \in \mathscr{F}_{h_{s}^{(2)}}\left(d^{(2)-1}(s), \underline{A}\right): f\left(v_{1}, v_{2}\right) \in A_{\underline{v}_{1}}\right\}
$$

and give $I^{(2)}(\underline{A})=\bigcup_{s \in S} I^{(2)}(\underline{A})_{s}$ the structure of a Polish field of groups. $R$ acts on $I^{(2)}(\underline{A})$ by

$$
T^{(2)}\left(v_{3}\right) F\left(v_{1}, v_{2}\right)=F\left(v_{1}, v_{2} v_{3}\right) \quad v_{1}, v_{2}, v_{3} \in R^{(3)} .
$$

This action makes $I^{(2)}(\underline{A})$ an $R$-module.

Proposition 2.5. There is a canonical isomorphism of $R$-modules

$$
I(I(\underline{A})) \cong I^{(2)}(\underline{A}) \text {. }
$$

Proof. The map is given by $K(s): I(I(\underline{A}))_{s} \rightarrow I^{(2)}(\underline{A})_{s},(K(s) F)\left(v_{1}, v_{2}\right)=$ $F\left(v_{2}\right)\left(v_{1}\right), v_{1}, v_{2} \in d^{(2)-1}(s)$. It is a routine check that this is a welldefined injective $R$-module map.

The surjectivity is essentially Proposition 2.4, after making the following identifications:

$$
\begin{aligned}
I(\underline{A})_{s} & \cong \mathscr{F}_{h_{s}}\left(d^{-1}(s), A_{s}\right) ; \quad f(v) \longmapsto J(v)^{-1} f(v) \\
I(I(\underline{A}))_{s} & \cong \mathscr{F}_{h_{s}}\left(d^{-1}(s), I(\underline{A})_{s}\right), \quad F(w) \longmapsto T(w)^{-1} F(w)
\end{aligned}
$$


and hence

$$
\begin{gathered}
I(I(\underline{A}))_{s} \stackrel{A}{\cong} \mathscr{F}_{h_{s}}\left(d^{-1}(s), \mathscr{F}_{h_{s}}\left(d^{-1}(s), A_{s}\right)\right) ; \\
(A F)(w)(v)=J(v)^{-1}\left[F(w)\left(v w^{-1}\right)\right]
\end{gathered}
$$

also

$$
\begin{gathered}
I^{(2)}(\underline{A})_{s} \stackrel{B}{\cong} \mathscr{F}_{s}^{(2)}\left(d^{(2)-1}(s), A_{s}\right) ; \\
B G(x, y)=J(x y)^{-1} G(x, y) \quad x, y \in R^{(2)}
\end{gathered}
$$

and $d^{(2)-1}(s), h_{s}^{(2)} \cong d^{-1}(s) \times d^{-1}(s), h_{s} \times h_{s}, p, q \longrightarrow p q, q$ (observe the last isomorphism preserves measure class by quasi-invariance of the measures $h_{s}$ ).

Define $C: \mathscr{F}_{h_{s}}\left(d^{-1}(s), \mathscr{F}_{h_{s}}\left(d^{-1}(s), A_{s}\right)\right) \rightarrow \mathscr{F}_{h_{s} \times h_{s}}\left(d^{-1}(s) \times d^{-1}(s), A_{s}\right)$ by $C(f)\left(v_{1}, v_{2}\right)=F\left(v_{1}\right)\left(v_{2}\right) . \quad C$ is an isomorphism by [6] Theorem 1. Then for $F \in I(I(\underline{A}))_{s}$,

$$
C A F=B K F
$$

Propostion 2.6 (c.f. [6] Proposition 15). There is a canonical isomorphism of $R$-modules $U(I(\underline{A})) \cong I(U(\underline{A}))$.

Proof. Both modules are quotients of $I(I(\underline{A}))$ which by Proposition 2.5 may be identified with $I^{(2)}(\underline{A})$. Under this identification, to obtain $U(I(\underline{A}))$ we factor by functions

$$
B_{1}=\left\{F\left(v_{1}, v_{2}\right)=\left[T\left(v_{2}\right) f\right]\left(v_{1}\right)=f\left(v_{1} v_{2}\right): f \in I(\underline{A})_{\underline{v_{1}}},\left(v_{1}, v_{2}\right) \in R^{(2)}\right\} .
$$

To obtain $I(U(\underline{A}))$, we factor by

$$
B_{2}=\left\{F\left(v_{1}, v_{2}\right)=J\left(v_{1}\right) a\left(v_{2}\right): a\left(v_{2}\right) \in A_{\underline{v}_{2}}\right\} .
$$

It is straightforward to check that $K: I^{(2)}(\underline{A}) \rightarrow I^{(2)}(\underline{A}), K F\left(v_{1}, v_{2}\right)=$ $J\left(v_{1}\right) F\left(v_{1}^{-1}, v_{1} v_{2}\right)$ is an isomorphism of $R$-modules which carries $B_{1}$ onto $B_{2}$.

We are now able to prove the vanishing axiom for $n>0$ :

Proposition 2.7 (c.f. [6] Proposition 22). For any $A$, and any $n>0$, the map $H^{n}(R, \underline{A}) \rightarrow H^{n}(R, I(\underline{A}))$ induced by the inclusion $\underline{A} \rightarrow I(\underline{A})$ is the zero map, and similarly for $\underline{H}$.

Proof. We prove the result for $\underline{H}^{n}$. It will be seen that the same proof works for $H^{n}$ with the omission of a.e. considerations. By Proposition 2.4, $L: \underline{C}^{n}(R, A) \rightarrow \underline{C}^{n-1}(R, I(\underline{A}))$, is an isomorphism. We also have an involution of

$$
\underline{C}^{n}(R, A), h \longmapsto h^{*}, h^{*}\left(v_{1}, \cdots, v_{n}\right)=J\left(\underline{v}_{1}, \bar{v}_{n}\right) h\left(v_{n}^{-1}, \cdots, v_{1}^{-1}\right)
$$


which preserves $\underline{B}^{n}$ and $\underline{Z}^{n}$; and a map

$$
\begin{aligned}
& T: \underline{C}^{n-1}(R, I(\underline{A})) \longrightarrow \underline{C}^{n-1}(R, I(\underline{A})), \\
& T f\left(v_{1}, \cdots, v_{n-1}\right)\left(v_{0}\right)=f\left(v_{n-1}^{-1}, \cdots, v_{1}^{-1}\right)\left(v_{0} v_{1} \cdots v_{n-1}\right) .
\end{aligned}
$$

Let $i: \underline{C}^{n}(R, \underline{A}) \rightarrow C^{n}(R, I(\underline{A}))$ be the map induced by the inclusion map. One may check that if $f \in \underline{Z}^{n}(R, \underline{A})$, then $\partial T L f^{*}=i(f)$.

An inductive argument using the long exact sequence of cohomology derived from $0 \rightarrow I(\underline{A}) \rightarrow I(I(\underline{A})) \rightarrow U(I(\underline{A})) \rightarrow 0$ together with Propositions 2.6 and 2.7 now reduces the question of the vanishing of $\underline{H}^{n}(R, I(\underline{A})), H^{n}(R, I(\underline{A}))$ to the case $n=1$. (c.f. [6] p. 22). This we check directly:

Proposition 2.8. $H^{1}(R, I(\underline{A}))=\underline{H}^{1}(R, I(\underline{A}))=0$.

Proof. First consider $\underline{H}^{1}$. Choose $F \in \underline{Z}^{1}(R, I(\underline{A}))$. Then

$$
0=T\left(v_{1}\right) F\left(v_{2}\right)-F\left(v_{1} v_{2}\right)+F\left(v_{1}\right) \text { a.a. }\left(v_{1}, v_{2}\right) \in R^{(2)} .
$$

Therefore, a.a. $\left(v_{1}, v_{2}\right) \in R^{(2)}$

$$
F\left(v_{2}\right)\left(v_{0} v_{1}\right)+F\left(v_{1}\right)\left(v_{0}\right)=F\left(v_{1} v_{2}\right)\left(v_{0}\right) \text { a.a. } v_{0} \in d^{-1}\left(\underline{v}_{1}\right),
$$

hence

$$
F\left(v_{2}\right)\left(v_{0} v_{1}\right)+F\left(v_{1}\right)\left(v_{0}\right)=F\left(v_{1} v_{2}\right)\left(v_{0}\right) \text { a.a. } v_{0}, v_{1}, v_{2} \in R^{(3)} .
$$

Setting $x=v_{0} v_{1}, y=v_{2}$ we obtain

$$
F(y)(x)=F\left(v_{0}^{-1} x y\right)\left(v_{0}\right)-F\left(v_{0}^{-1} x\right)\left(v_{0}\right) \quad \text { a.a. } v_{0}^{-1}, x, y \in R^{(3)} .
$$

Therefore, for a.a. $s \in S, \exists v_{0} \in g^{-1}(s)$ such that (3) holds a.a. $x, y \in$ $g^{(2)-1}(s)$. We may choose $v_{0}(s)$ such that $s \mapsto v_{0}(s)$ is Borel. Define $G \in C^{0}(R, I(\underline{A}))$,

$$
\begin{aligned}
G(s)(w)= & F\left(v_{0}(\underline{w})^{-1} w\right)\left(v_{0}(\underline{w})\right) \\
\partial G(v)(w)= & T(v) G(\bar{v})(w)-G(\underline{v})(w)=F\left(v_{0}(\underline{w})^{-1} w v\right)\left(V_{0}(\underline{w})\right) \\
& -F\left(v_{0}(\underline{w})^{-1} w\right)\left(v_{0}(\underline{w})\right)=F(v)(w) \text { a.a. }(w, v) \in R^{(2)} .
\end{aligned}
$$

Hence $F=\partial G$ in $\underline{C}^{1}(R, I(\underline{A}))$, so $\underline{H}^{1}(R, I(\underline{A}))=0$.

It is clear that a similar argument, with the omission of a.e. qualifications, shows that $H^{1}(R, I(\underline{A}))=0$.

In conclusion we have

TheOREm 2.9. The natural maps $H^{n}(R, A) \rightarrow \underline{H}^{n}(R, A)$ are isomorphisms in all dimensions. 
3. Groupoid extensions. Let $R$ be a principal groupoid with unit space $S$ and let $\Gamma$ be a measured field of l.c.s.c. groups on $S$. A groupoid $\mathscr{G}$ is an extension of $R$ by $\Gamma$ if $R \mathscr{G}=R$ and $\Gamma \mathscr{G}=\Gamma$. This terminology is of course motivated by Theorem 1.1.

An abstract kernel is a triple $(R, \Gamma, J)$ where $R, \Gamma$ are as above and, for a.a. $v \in R, J(v): \Gamma_{\bar{v}} \rightarrow \Gamma_{\underline{v}}$ is a topological isomorphism such that a.e. $(u, v) \in R^{(2)}, J(u v)^{-1} J(u) J(v) \in \operatorname{Int} \Gamma_{\bar{v}}$, and such that the map $R_{d, p}^{*} \Gamma \rightarrow \Gamma,(v, \xi) \rightarrow J(v) \xi$, is Borel. A morphism of abstract kernels $\mathrm{t}:(R, \Gamma, J) \rightarrow\left(R^{\prime}, \Gamma^{\prime}, J^{\prime}\right)$ consists of

(i) A groupoid homomorphism $\tau: R \rightarrow R^{\prime}$.

(ii) A Borel homomorphism $\underline{T}: \Gamma \rightarrow \Gamma^{\prime}$ over $\tau$, i.e., a field of maps $T_{s}: \Gamma_{s} \rightarrow \Gamma_{\tau s}^{\prime}$, such that

$$
T_{\underline{u}} J(u)=\operatorname{Ad} \beta(u) J^{\prime}(\tau(u)) T_{\bar{u}} \quad \text { a.e. } u \in R,
$$

where $\beta(u) \in \Gamma_{\tau(\underline{u})}$, and $\operatorname{Ad} x(y)=x^{-1} y x$.

Morphisms $\mathrm{t}, \mathrm{t}^{\prime}$ are conjugate if $T_{s} \in T_{s}^{\prime} \operatorname{Int} \Gamma_{s} \forall s \in S$.

Proposition 3.1. There is a functor $\mathfrak{A}$ from the category of groupoids and homomorphisms to the category of abstract kernels and morphisms.

Proof. Let $\mathscr{G}$ be a groupoid. Set $R=R \mathscr{G}$ and $\Gamma=\Gamma \mathscr{G}$. Let $v \mapsto \alpha(v)$ be a Borel map $R \rightarrow \mathscr{G}$ such that $P(\alpha(v))=v$ a.a. $v \in R$. Define $J(v): \Gamma_{\bar{v}} \rightarrow \Gamma_{\underline{v}}, J(v)(\xi)=\alpha(v) \xi \alpha(v)^{-1}$. It is straightforward to check that $\mathfrak{A}(\mathscr{G})=(R, \Gamma, J)$ is an abstract kernel and that different choices $\alpha^{\prime}, J^{\prime}$ give isomorphic kernels. Let $\pi: \mathscr{G} \rightarrow \mathscr{G}^{\prime}$ be a homomorphism. $\pi$ induces $\tau^{\pi}: R \mathscr{G} \rightarrow R \mathscr{G}^{\prime}$ and a homomorphism $T^{\pi}: \Gamma \mathscr{G} \rightarrow$ $\Gamma \mathscr{G}^{\prime}$ by restriction, $T_{s}^{\pi}$ being Borel and hence continuous by [1] $\mathrm{p}$. 23. Also

$$
T_{\underline{u}}^{\pi} J(u)=\operatorname{Ad}\left(\alpha(\tau(u)) \pi(\alpha(u))^{-1}\right) J^{\prime}(\tau u) T_{\bar{u}}^{\pi} \quad \forall u \in R
$$

and

$$
\alpha(\tau(u)) \pi(\alpha(u))^{-1} \in \Gamma_{\underline{\tau(u)}}
$$

so that we have a morphism $\mathfrak{A}(\pi): \mathfrak{A}(\mathscr{G}) \rightarrow \mathfrak{A}\left(\mathscr{G}^{\prime}\right)$.

It is clear that this correspondence is functorial.

If $\mathscr{G}$ is a groupoid and $\mathfrak{A}(\mathscr{G})=(R, \Gamma, J)$ then clearly $\mathscr{G}$ is an extension of $\Gamma$ by $R$. We now investigate the extent to which an abstract kernel $(R, \Gamma, J)$ determines all groupoid extensions of $\Gamma$ by $R$.

By von Neumann's selection lemma, an abstract kernel gives a function $f \in \mathscr{F}_{h(2)}\left(R^{(2)}, \Gamma\right), f(u, v) \in \Gamma_{\underline{u}}$, such that

$$
J(u) J(v)=A d f(u, v) J(u v) \text { a.a. } u, v \in R^{(2)} .
$$


Hence a.a. $u, v, w \in R^{(3)}$ :

$$
\begin{aligned}
J(u v w) & =\operatorname{Ad} f(u v, w)^{-1} J(u v) J(w)=\operatorname{Ad} f(u v, w)^{-1} \operatorname{Ad} f(u, v)^{-1} J(u) J(v) J(w) \\
J(u v w) & =\operatorname{Ad} f(u, v w)^{-1} J(u) J(v w) \\
& =\operatorname{Ad} f(u, v w)^{-1} J(u) \operatorname{Ad} f(v, w)^{-1} J(u)^{-1} J(u) J(v) J(w)
\end{aligned}
$$

so that

$$
f(u v, w) f(u, v)=k(u, v, w) f(u, v w) J(u) f(v, w)
$$

where $k(u, v, w) \in Z\left(\Gamma_{\underline{u}}\right)$, the center of $\Gamma_{\underline{u}}$, a.a. $u, v, w \in R^{(3)}$.

LEMмA 3.2 (c.f. [5], Lemma 8.4, Ch. IV). $k \in \underline{Z}^{3}(R, Z(\Gamma))$ where $Z(\Gamma)$ is the field given by $Z(\Gamma)_{s}=Z\left(\Gamma_{s}\right)$.

Proof. Pick $t, u, v, w \in R^{(4)}$. Set $L=J(t)[f(u, v w) J(u) f(v, w)]$. For a.a. $t, u, v, w \in R^{(4)}$, we may expand $L$ in two ways using (A) and $(B)$ repeatedly:

$$
\begin{aligned}
L= & J(t) f(u, v w) J(t) J(u) f(v, w) \\
= & f(t, u v w)^{-1} f(t u, v w) f(t, u) k(t, u, v w)^{-1} \operatorname{Ad} f(t, u)[J(t u) f(v, w)] \\
= & f(t, u v w)^{-1} f(t u, v w) f(t u, v w)^{-1} f(t u v, w) f(t u, v) f(t, u) k(t u, v, w)^{-1} \\
& \times k(t, u, v w)^{-1}
\end{aligned}
$$

and

$$
\begin{aligned}
L= & J(t)\left[f(u v, w) f(u, v) k(u, v, w)^{-1}\right] \\
= & f(t, u v w)^{-1} f(t u v, w) f(t, u v) f(t, u v)^{-1} f(t u, v) f(t, u) k(t, u v, w)^{-1} \\
& \times k(t, u, v)^{-1} J(t) k(u, v, w)^{-1} .
\end{aligned}
$$

Comparing these expressions one sees $k \in \underline{Z}^{3}$.

LEMma 3.3. The image of $k$ in $\underline{H}^{3}$ is independent of the choice of $f$ and every $k^{\prime}$ in the class of $k$ is obtained for some choice of $f$.

Proof. This is routine to check following the lines of [5] Lemma 8.5, Chapter IV.

We write $o b s(R, \Gamma, J)$ for the image of $k$ in $\underline{H}^{3}$. This is further justified by

LEMma 3.4. The image of $k$ in $\underline{H}^{3}(R, Z(\Gamma))$ depends only on the isomorphism class of $(R, \Gamma, J)$.

Proof. First observe that an isomorphism $(R, \Gamma, J) \rightarrow\left(R^{\prime}, \Gamma^{\prime}, J^{\prime}\right)$ induces an isomorphism $\underline{H}^{*}(R, Z(\Gamma)) \rightarrow \underline{H}^{*}\left(R^{\prime}, Z\left(\Gamma^{\prime}\right)\right)$ in an obvious way. 
Since $T_{\underline{u}} J(u) T_{\bar{u}}^{-1}=\operatorname{Ad} \beta(u) J^{\prime}(u), \beta(u) \in \Gamma_{\underline{u}}^{\prime}$, a.a. $u \in R$, we obtain using (A)

$$
J^{\prime}(u) J^{\prime}(v)=\operatorname{Ad} f^{\prime}(u, v) J^{\prime}(u v) \quad \text { a.a. } u, v \in R^{(2)}
$$

where $f^{\prime}(u, v)=\beta(u v)\left(T_{\underline{u}} f(u, v)\right)\left(T_{\underline{u}} J(u) T_{\bar{u}}^{-1} \beta(v)^{-1}\right) \beta(u)^{-1}$. Thus $f^{\prime}, J^{\prime}$ satisfy $(\mathrm{A})$.

Now set $K_{1}=f^{\prime}(u v, w) f^{\prime}(u, v) ; K_{2}=f^{\prime}(u, v w) J^{\prime}(u) f^{\prime}(v, w)$.

Using (A) one computes

$$
\begin{aligned}
K_{1}= & \beta(u v w)\left(T_{\underline{u}} f(u v, w)\right) T_{\underline{u}}\left(f(u, v) J(u) J(v) T_{\bar{v}}^{-1} \beta(w)^{-1}\right)\left(T_{\underline{u}} J(u) T_{\bar{u}}^{-1} \beta(v)^{-1}\right) \\
& \times \beta(u)^{-1} \\
K_{2}= & \beta(u v w)\left(T_{\underline{u}} f(u, v w)\right)\left(T_{\underline{u}} J(u) f(v, w)\right)\left(T_{\underline{u}} J(u) J(v) T_{\bar{v}}^{-1} \beta(w)^{-1}\right) \\
& \times\left(T_{\underline{u}} J(u) T_{\bar{u}}^{-1} \beta(v)^{-1}\right) \beta(u)^{-1}
\end{aligned}
$$

and so

$$
k^{\prime}(u, v, w)=K_{1} K_{2}^{-1}=T_{\underline{u}} k(u, v, w) .
$$

THEOREM 3.5. Let $(R, \Gamma, J)$ be an abstract kernel and suppose that obs $(R, \Gamma, J)=0$. Then the extensions of $\Gamma$ by $R$ are in one-one correspondence with $\underline{H}^{2}(R, Z(\Gamma))$.

Proof. By Lemma 3.4 we may suppose that $k=0$ a.e. Set $\mathscr{G}=$ $R_{g, p}^{*} \Gamma$ with the fiber product measure $\omega$. Define, for $u, v \in R^{(2)}, a \in$ $\Gamma_{\underline{u}}, b \in \Gamma_{\underline{v}},\left(\overline{u, a)}=\bar{u} ;(\underline{u, a})=\underline{u} ;(u, a)(v, b)=\left(u v, a(J(u) b) f(u, v)^{-1}\right)\right.$; $(u, a)^{-1}=\left(u^{-1}, f\left(u^{-1}, u\right) J\left(u^{-1}\right) a^{-1}\right)$. Using (A) and (B), one checks that these definitions of multiplication and inversion satisfy the appropriate identities almost everywhere. $\omega$ also has appropriate invariance properties: the fiber measure on $F^{\overline{u, a}}=F^{\bar{u}} \times \Gamma_{\bar{u}}$ is $\omega^{\overline{u, a}}=$ $h^{\bar{u}} \times \nu_{\bar{u}}$, where $\nu_{s}$ is the measure on $\Gamma_{s} . \quad T^{u, a}: F^{\overline{u, a}} \rightarrow F^{u, a}$ is $T^{u, a}(v, b)=$ $\left(u v, a(J(u) b) f(u, v)^{-1}\right)$. By the quasi-invariance of the measures $h^{s}$ and the fact that automorphisms of $\Gamma_{s}$ preserve $\left[\nu_{s}\right]$, the measure $\omega^{\overline{u, a}}$ are also quasi-invariant. For $v \in R$, set $P_{v}: \Gamma_{\underline{v}} \rightarrow \Gamma_{\bar{v}}, P_{v}(a)=$ $f\left(v^{-1}, v\right) J\left(v^{-1}\right) a^{-1}$. Then $P_{v^{*} \nu_{\underline{v}}} \sim \nu_{\bar{v}}$ and

$$
i_{*} \omega=i_{*} \int_{R} \nu_{\underline{v}} d h(v)=\int_{R} P_{v} * \nu_{\underline{v}} d h(v) \sim \int_{R} \nu_{\bar{v}} d h(v) \sim \int_{R} \nu_{\underline{v}} d h(v)=\omega .
$$

$\mathscr{G}$ is therefore almost a groupoid, and we wish to conclude the existence of a groupoid $\hat{\mathscr{G}}$ which is almost equal to $\mathscr{G}$, in a suitable sense. But this is precisely the content of [8], Theorem 3.7; namely, there is a groupoid $\hat{\mathscr{G}}$ and a map $j: \mathscr{G} \rightarrow \hat{\mathscr{G}}$ which is almost a groupoid homomorphism. We write $\mathscr{G}=\operatorname{Ext}_{f}(R, \Gamma), \hat{\mathscr{G}}=\widehat{\operatorname{Ext}}_{f}(R, \Gamma)$.

Suppose now that $f$ is replaced by a function $f^{\prime}$, so that $f^{\prime}(u, v)=$ 
$f(u, v) h(u, v)$ a.a. $(u, v) \in R^{(2)}$, where $h(u, v) \in \underline{Z}^{2}(R, Z(\Gamma))$. It is clear that any extension of $\Gamma$ by $R$ may be obtained in this way. If $h \in$ $\underline{B}^{2}(R, Z(\Gamma)), h=\partial g$, then $P: \operatorname{Ext}_{f}(R, \Gamma) \rightarrow \operatorname{Ext}_{f^{\prime}}(R, \Gamma), P(u, a)=$ $(u, g(u) a)$, is an almost isomorphism (i.e., it satisfies the appropriate conditions a.e.) and hence induces an equivalence $\hat{P}: \widehat{\operatorname{Ext}}_{f}(R, \Gamma) \rightarrow$ $\widehat{\operatorname{Ext}}_{f}(R, \Gamma)$. ([8] Theorem 4.1.)

Conversely suppose that $\widehat{\operatorname{Ext}}_{f}(R, \Gamma)$ and $\widehat{\mathrm{Ext}}_{f},(R, \Gamma)$ are equivalent extensions of $\Gamma$ by $R$, via an isomorphism $\hat{T}$. Then there is an almost isomorphism $T: \operatorname{Ext}_{f}(R, \Gamma) \rightarrow \operatorname{Ext}_{f^{\prime}}(R, \Gamma), T(u, a)=(u, P(u) a)$ where $P(s)=i d$ and $P(u): \Gamma_{u} \rightarrow \Gamma_{\underline{u}}$ is almost a bijection. The condition that $T$ is almost a homomorphism leads to

$$
\begin{aligned}
(P(u) a)(J(u) P(v) b) f^{\prime}(u, v)^{-1}= & P(u v)\left(a(J(u) b) f(u, v)^{-1}\right) \\
& \text { a.a. } u, v \in R^{(2)}, a \in \Gamma_{\underline{u}}, b \in \Gamma_{\underline{v}} .
\end{aligned}
$$

Setting $u=\bar{u},\left(T_{\underline{v}} a\right)(P(v) b)=P(v)(a b)$ so that a.a. $v, P(v) T_{\underline{v}}^{-1}$ commutes with all left multiplications in $\Gamma_{\underline{v}}$, and hence is right multiplication by an element $g(v) \in \Gamma_{\underline{v}}$. Setting $v=\underline{v},(P(u) a)\left(J(u) T_{\bar{u}} b\right)=P(u)(a(J(u) b)$. Therefore $P(u) T_{\underline{u}}^{-1}$ is also left multiplication by $g(u) \in \Gamma_{\underline{u}}$, a.a. $u$.

We conclude $g(u) \in Z\left(\Gamma_{u}\right)$ a.a. $u$, and substituting back in (1) leads to

$$
g(u)(J(u) g(v)) f^{\prime}(u, v)^{-1}=g(u v) f(u, v)^{-1}
$$

so.that $h=\partial g \in \underline{B}^{2}(R, Z(\Gamma))$.

From now on we will write $\operatorname{Ext}_{J}(R, \Gamma)$ for $\widehat{\operatorname{Ext}_{f}}(R, \Gamma)$.

We now return to the special abstract kernels associated to groupoids.

Proposition 3.6. If $(R, \Gamma, J)=\mathfrak{2}(\mathscr{G})$, then obs $(R, \Gamma, J)=0$.

Proof. By the definition of $\mathfrak{A}(\mathscr{G})$, we have a Borel map $\alpha: R \rightarrow$ $\mathscr{G}, P(\alpha(u))=u$ a.a. $u \in R$, and

$$
J(u)(\xi)=\alpha(u) \xi \alpha(u)^{-1}, \quad \xi \in \Gamma_{\bar{u}} .
$$

Now $\alpha(u v) \alpha(v)^{-1} \alpha(u)^{-1} \in \Gamma_{u}$ a.a. $u, v \in R^{(2)}$. Set

$$
f(u, v)=\alpha(u v) \alpha(v)^{-1} \alpha(u)^{-1} .
$$

It is easy to compute that $f$ satisfies (A) and (B) with $k=0$.

Proposition 3.7. Let $(R, \Gamma, J)=\mathfrak{A}(\mathscr{G})$, and let $f$ be such that (A), (B) hold with $k=0$. Then $\operatorname{Ext}_{J}(R, \Gamma)$ and $\mathscr{G}$ are equivalent extensions of $\Gamma$ by $R$. 
Proof. The map $\xi \mapsto\left(P(\xi), \alpha(P(\xi))^{-1} \xi\right)$ is an almost isomorphism.

We have shown that groupoid homomorphisms induce morphisms of abstract kernels. We now investigate whether a morphism $\mathrm{t}:(R, \Gamma, J) \rightarrow(\bar{R}, \bar{\Gamma}, \bar{J})$ determines a homomorphism $\operatorname{Ext}_{J}(R, \Gamma) \rightarrow$ $\operatorname{Ext}_{\bar{J}}(\bar{R}, \bar{\Gamma})$, and discuss the similarity of such morphisms.

Suppose that $o b s(R, \Gamma, J)=o b s(\bar{R}, \bar{\Gamma}, \bar{J})=0$ and that $f, \bar{f}$ are chosen so that (A), (B) hold with $k, \bar{k}=0$. By definition we have a homomorphism $\tau: R \rightarrow \bar{R}$ and a field of homomorphisms $T_{s}$ over $\tau$ such that

$$
T_{\underline{u}} J(u)=\operatorname{Ad} \beta(u) \bar{J}(\tau u) T_{\bar{u}}, \quad \beta(u) \in \bar{\Gamma}_{\underline{\tau(u)}} .
$$

An almost homomorphism $\operatorname{Ext}(R, \Gamma) \rightarrow \operatorname{Ext}(\bar{R}, \bar{\Gamma})$ consistent with $t$ must be of the form

$$
(u, a) \longmapsto(\tau u, H(u) a) \text { a.a. }(u, a) \in \operatorname{Ext}(R, \Gamma),
$$

where $H(u): \Gamma_{\underline{u}} \rightarrow \bar{\Gamma}_{\tau \underline{u}}$ and $H(\underline{u})=T_{\underline{u}}$.

The condition that this be an almost homomorphism is

$$
H(u v)\left(a(J(u) b) f(u, v)^{-1}\right)=(H(u) a) \bar{J}(\tau u)(T(\bar{u}) b) \bar{f}(\tau u, \tau v)^{-1} \text { a.e. }
$$

Let $h(u)=H(u)$. Putting $v=\underline{v}, \alpha=e$ in (3.2) gives

$$
H(u)(c)=h(u) \beta(u) T_{\underline{u}} c \beta(u)^{-1}, \quad c \in \Gamma_{\underline{u}} .
$$

Putting $u=\bar{u}, b=e$ gives

$$
H(v)(a)=\left(T_{\underline{v}} a\right) h(v) .
$$

Comparing these equations we see that

$$
h(v) \beta(v) \in Z\left(T_{\underline{v}} \Gamma_{\underline{v}}\right) \quad \text { a.a. } v \in R
$$

and

$$
H(v)(\alpha)=\left(T_{\underline{v}} a\right) h(v) \text { a.a. } v \in R .
$$

Substituting back in (3.2) gives

$$
T_{\underline{u}} f(u, v)^{-1} h(u v)=h(u)(\bar{J}(\tau u) h(v)) \bar{f}(\tau u, \tau v)^{-1} .
$$

Now using (A), (3.1) and (3.5) we obtain

$$
\operatorname{Ad}\left(\bar{f}(\tau u, \tau v) \bar{J}(\tau u) h(v)^{-1} h(u)^{-1}\right) T_{\underline{v}}=\operatorname{Ad}\left(h(u v)^{-1} T_{\underline{u}} f(u, v)\right) T_{\underline{v}}
$$




$$
\bar{f}(\tau u, \tau v) \bar{J}(\tau u) h(v)^{-1} h(u)^{-1} T_{\underline{u}} f(u, v)^{-1} h(u v) \in Z\left(T_{\underline{v}}\left(\Gamma_{\underline{v}}\right)\right) .
$$

Therefore $\mathrm{t}$ induces a homomorphism $\operatorname{Ext}_{J}(R, \Gamma) \rightarrow \operatorname{Ext}_{\bar{J}}(\bar{R}, \bar{\Gamma})$ precisely when there is a choice of $h(u) \in \bar{\Gamma}_{\underline{\underline{\tau}}}$ such that

$$
\operatorname{Ad} h(u)=\operatorname{Ad} \beta(u)^{-1} \text { a.e. }
$$

and (3.7) holds a.e., moreover (3.9) follows from (3.10).

In special cases these conditions simplify: if $Z\left(T_{\underline{v}}\left(\Gamma_{\underline{v}}\right)\right)=\{e\}$ a.e. then $t$ always induces a homomorphism Ext $t$; if $T_{\underline{v}}$ has dense range a.e. one may compute that there is an obstruction to Ext $t$ being a homomorphism which is an element of $\underline{H}^{2}(R, Z(\bar{\Gamma}))$. If $\Gamma, \bar{\Gamma}$ are fields of abelian groups all obstructions vanish.

In a similar vein we investigate the similarity of morphisms t, $\mathrm{t}^{\prime}:(R, \Gamma, J) \rightarrow(\bar{R}, \bar{\Gamma}, \bar{J})$. Consider the special case $(R, \Gamma, J)=\mathfrak{A}(\mathscr{G})$, $(\bar{R}, \bar{\Gamma}, \bar{J})=\mathfrak{A}(\overline{\mathscr{G}}), \underline{T}=\mathfrak{A}(\pi), \underline{\bar{T}}^{\prime}=\mathfrak{A}\left(\pi^{\prime}\right)$, where $\pi, \pi^{\prime}$ are similar homomorphisms $\mathscr{G} \rightarrow \mathscr{G}$. Let $f, \bar{f}$ be functions such that (A), (B) hold a.e. with $k, \bar{k}=0$. There is a map $\Phi: S_{\mathscr{S}} \rightarrow \overline{\mathscr{G}}$,

$$
\Phi(\underline{\xi}) \pi^{\prime}(\xi) \Phi(\bar{\xi})^{-1}=\pi(\xi) \text { a.a. } \xi \in \mathscr{G} .
$$

Let $\tau, \tau^{\prime}$ and $\underline{T}, \underline{T}^{\prime}$ be the maps induced on $R, \Gamma$ by $\pi$ and $\pi^{\prime}$, and set $\theta(s)=\left(\tau s, \tau^{\prime} s\right) \in \bar{R}$. Then

$$
\bar{J}(\theta(s))=\operatorname{Ad} \Phi(s)^{-1} \operatorname{Ad} \gamma(s) \text { for some } \gamma(s) \in \Gamma_{\tau^{\prime} s} .
$$

In order to simplify computations we will replace $\pi^{\prime}$ by $\gamma(\underline{u}) \pi^{\prime}(u) \gamma(\bar{u})^{-1}$ which induces $\tau^{\prime}$ and a homomorphism conjugate to $\underline{T}^{\prime}$. Thus we assume $\gamma(s)=e$.

Applying (3.11) to $\Gamma_{s}$ gives

$$
T_{s}=\bar{J}(\theta(s)) T_{s}^{\prime} \quad \text { a.e. }
$$

Using the relations

$$
\begin{gathered}
T_{\underline{u}} J(u)=\operatorname{Ad} \beta(u) \bar{J}(\tau u) T_{\bar{u}} \\
J(u)=\operatorname{Ad} \alpha(u)^{-1}
\end{gathered}
$$

we obtain

$$
\operatorname{Ad} \beta(u) \bar{J}(\tau u) T_{\bar{u}}=\operatorname{Ad} \pi(\alpha(u))^{-1} T_{\bar{u}}
$$

and a similar relation $(3.15)^{\prime}$ for $T^{\prime}$.

Apply Ad to both sides of (3.11) and using (3.12), (3.13), (3.15):

$$
\operatorname{Ad} \beta(u) \bar{J}(\tau u) J(\theta(\bar{u})) \bar{T}_{\bar{u}}^{\prime}=J(\theta(\underline{u})) \operatorname{Ad} \beta^{\prime}(u) J\left(\tau^{\prime} u\right) T_{\bar{u}}^{\prime} \quad \text { a.e. }
$$

Definition 3.8. Morphisms $\mathrm{t}, \mathrm{t}^{\prime}:(R, \Gamma, J) \rightarrow(\bar{R}, \bar{\Gamma}, \bar{J})$ are similar 
if $\tau$ is similar to $\tau^{\prime}$ and $\mathrm{t}^{\prime}$ is conjugate to a morphism for which (3.12) and (3.16) hold.

Suppose now $\mathrm{t}, \mathrm{t}^{\prime}$ are similar, chosen such that (3.12) and (3.16) hold, and that both induce homomorphisms of $\operatorname{Ext}(R, \Gamma)$. There is an obstruction to Extt, Ext $t^{\prime}$ being similar, which we outline below.

From (3.1.6) and (A) we obtain

$$
\begin{aligned}
& \operatorname{Ad} \beta(u) \operatorname{Ad} \bar{f}(\tau u, \theta(\bar{u})) J(\tau u \theta(\bar{u})) T_{\bar{u}}^{\prime} \\
& \quad=\operatorname{Ad} J(\theta(\underline{u})) \beta^{\prime}(u) \operatorname{Ad} f\left(\theta(\underline{u}), \tau^{\prime} u\right) J\left(\theta(\underline{u}) \tau^{\prime} u\right) T_{\bar{u}}^{\prime}
\end{aligned}
$$

and hence

$$
\bar{f}(\tau u, \theta(\bar{u})) \beta(u)=\bar{f}\left(\theta(\underline{u}), \tau^{\prime} u\right)\left(J(\theta(\underline{u})) \beta^{\prime}(u)\right) l(u), l \in Z\left(T_{\underline{u}} \Gamma_{\underline{u}}\right) \quad \text { a.e. }
$$

A routine computation shows that if there is a function $\Phi$ such that

$$
\Phi(u) \operatorname{Ext} \mathrm{t}(u, a) \Phi(\bar{u})^{-1}=\operatorname{Ext} \mathrm{t}^{\prime}(u, \text { a) a.e. }
$$

then $\Phi(\underline{u})$ must be of the form $(\theta(\underline{u}), e)$ and that this is a similarity if we can choose $l(u)=e$ in (3.18).

Again if $Z\left(T_{\underline{u}} \Gamma_{\underline{u}}\right)=\{e\}$ a.e., $\Phi$ is necessarily a similarity. If $T_{\underline{u}}$ has dense range a.a., then $l(u) \in Z\left(\bar{\Gamma}_{\underline{u}}\right)$. Substituting the expression for $l$ obtained in (3.18) into the equation (3.7) for $t$, and using $(3.7)^{\prime}$ for $\mathrm{t}^{\prime}$ we find by repeated use of (A) and (B) that $l(u) \bar{J}(\tau u) l(v)=l(u v)$ so that replacing $\beta(u)$ by $\beta(u) l(u)^{-1}$ produces no change in any of the relations used except that the $l(u)$ term in (3.18) vanishes, so that Ext $t$ and Ext $t^{\prime}$ are indeed similar.

\section{Examples and applications.}

(A) Theorem 2.9 applied to $H^{1}(R, A)$ shows that if $\pi: R \rightarrow A$ is an almost everywhere cocycle, then there is a saturated conull set $S^{*} \subseteq S$, and a strict cocycle $\pi^{\prime}: R \cap S^{*} \times S^{*} \rightarrow A$, so that $\pi=\pi^{\prime}+\partial g$ in $\underline{C}^{1}(R, A)$. Since $\partial g$ is automatically a strict cocycle (we may clearly assume $g$ is Borel and everywhere defined on $S$ ), we may include $\partial g$ in $\pi^{\prime}$ and conclude the existence of a strict cocycle defined on a saturated conull set of $S$, equal to $\pi$ a.e. This is a much stronger result than [7] Theorem 5.1, in which $\pi^{\prime}$ is only asserted to be strict on an i.c. of $R$. This fact simplifies the proof of many results on cocycles on $G$ spaces, for example the construction of the range closure of $\pi: S \times G \rightarrow A$. It may be seen by following through the steps of the proof that the result in fact depends neither on the freeness of the $G$ action nor on the commutativity of $A$. (This result was first observed and proved directly by L. Brown, private communication.) 
(B) Split extensions. An extension $\operatorname{Ext}_{J}(R, \Gamma)$ of $R$ by $\Gamma$ is said to be split if we can choose $f=0$ in (A). In this case $J: R \rightarrow$ Aut $\Gamma$ is a cocycle, where Aut $\Gamma$ is an algebraic groupoid in an obvious way. It is not hard to see that $\operatorname{Ext}_{J}(R, \Gamma)$ depends up to isomorphism on the cohomology class of $J$ in Aut $\Gamma /$ Int $\Gamma$.

Let $\tau: R^{\prime} \rightarrow R$ be a homomorphism of principal groupoids, and let $\underline{T}: \Gamma^{\prime} \rightarrow \Gamma$ be a morphism of the fields $\Gamma^{\prime}, \Gamma$ on $S^{\prime}, S$ over $\tau$, such that each $T_{s}$ is an isomorphism. If $J: R \rightarrow$ Aut $\Gamma$ is a cocycle, then $J^{T,=}(u)=T_{\underline{u}}^{-1} J(\tau(u)) T_{\bar{u}}, u \in R^{\prime}$, is a cocycle $R^{\prime} \rightarrow$ Aut $\Gamma^{\prime}$. In particular if $\tau$ is a similarity then $\operatorname{Ext}_{J}(R, \Gamma)$ and $\operatorname{Ext}_{J},:\left(R^{\prime}, \Gamma^{\prime}\right)$ are similar.

Conversely let $\pi: \operatorname{Ext}_{J}\left(R^{\prime}, \Gamma^{\prime}\right) \rightarrow \operatorname{Ext}_{J}(R, \Gamma)$ be a similarity with inverse $\psi$, so that $\psi \circ \pi$ is similar to the identity. Then, as in Proposition 3.1, $\tau^{\pi}: R^{\prime} \rightarrow R$ is a similarity. Moreover applying 3.12 to $\underline{T}^{\psi} \circ \underline{T}^{\pi}$ we see that each $T_{s}^{\pi}$ is an isomorphism. By $(3.1), T_{\underline{u}}^{\pi} J^{\prime}(u) T_{u}^{\tilde{\tau}-1}=$ Ad $k(u) J\left(\tau^{\prime} u\right), u \in R^{\prime}$, where $k(u) \in \Gamma_{\tau^{\pi}(u)}$. Therefore

$$
J^{\prime}(u)=\operatorname{Ad}\left(T_{\underline{u}}^{\pi-1} k(u)\right) J^{T^{\tilde{*}},-\bar{\prime}}(u),
$$

so that $J^{\prime}=J^{T^{\pi}, \sigma^{*}}$ up to a cocycle in Int $\Gamma^{\prime}$.

Hence the similarity classes of split extensions of $R$ by $\Gamma$ correspond to cohomology classes of cocycles $J: R \rightarrow$ Aut $\Gamma /$ Int $\Gamma$. In particular, taking $\Gamma$ to be a constant field $H$ and $R$ to be a hyperfinite relation, one obtains many dissimilar split extensions of $R$ by $H$.

(C) Following the methods of [2] Theorem 5, one shows that if an equivalence relation $R$ is generated by a free action of a l.c.s.c group $G$ on $S$, then $H^{*}(R, \underline{A})=H^{*}(G, \mathscr{F}(S, \underline{A}))$. In particular if $R$ is hyperfinite or if $G$ is a free group, then $H^{n}(R, \underline{A})=0$ for $n \geqq 2$ (c.f. [2] Theorem 6) and we may construct $\operatorname{Ext}_{f}(R, \Gamma)$ for any field $\Gamma$ over $S$. If $\Gamma$ is a field of abelian groups we may in addition choose $f=0$ so that the extension splits.

(D) It is natural to ask whether all the extensions we construct are similar to groupoids coming from group actions. Groupoids are countably similar if they are similar under mappings which are countable to one on the units, c.f. [3]. We have the following partial result:

Proposition 4.1. Let $R$ be a hyperfinite equivalence relation and let $H$ be a l.c.s.c. group and $J: R \rightarrow$ Aut $H$ a Borel cocycle. Then there is a l.c.s.c. group $G$ and a standard Borel $G$ space $X$ so that $X \times G$ is countably similar to $\operatorname{Ext}_{J}(R, H)$.

Proof. It is well known that $J$ may be replaced by a similar cocycle $J^{\prime}$ taking values in a countable subgroup $D$ of Aut $H$. (This uses hyperfiniteness of $R$ and the fact that Aut $\mathrm{H}$ is separable in 
the compact open topology, c.f. e.g. [11] Corollary 9.2). Realize $R$ as generated by a transformation $T$ on $S$ and form $X=S \times D$. On $X$ we have the skew action $T^{J}$ and the translation action of $D$ on itself. These two actions together generate a relation $R^{\prime}$ countably similar to $R . J^{\prime}\left(s, d ; s^{\prime}, d^{\prime}\right)=d+J\left(s, s^{\prime}\right)-d^{\prime}$ is a cocycle on $R^{\prime}$ similar to $J$ on $R$. Let $G=H(S)(Z \times D)$ where the $Z \times D$ action on $H$ is $(n, d) \cdot h=d(h)$. Let $G$ act on $X, H$ acting trivially and $\boldsymbol{Z} \times D$ as described above. Then $\operatorname{Ext}_{J}\left(R^{\prime}, H\right)=X \times G$, and by (B) $\operatorname{Ext}_{J}\left(R^{\prime}, H\right)$ is countably similar to $\operatorname{Ext}_{J}(R, H)$. For one computes that $J^{\prime}(x, x g)=p(g)$, where $p: G \rightarrow D$ is projection, so that $J(x, x g)=$ Ad $g: H \rightarrow H \quad \forall x \in X, g \in G$.

On the other hand, an example of Hahn [3] shows that there is a field of l.c.s.c. groups $\Gamma$ on a hyperfinite relation $R$, and a cocycle $J: R \rightarrow$ Aut $\Gamma$, such that $\operatorname{Ext}_{J}(R, \Gamma)$ is not countably similar to any $X \times G$.

(E) Remark that the groupoid $G^{\sigma}$ described in [10] $\S 7$ is a special case of our constructions.

\section{REFERENCES}

1. S. Banach, Théorie des opérations linéaries, Monografie Mat., PWN, Warsaw, 1932; reprint, Chelsea, New York, 1955.

2. J. Feldman and C. C. Moore, Ergodic equivalence relations, cohomology, and von Neumann algebras, I, Trans. Amer. Math. Soc., 234 (1977), 289-324.

3. J. Feldman, P. Hahn and C. C. Moore, Orbit structure and countable sections for actions of continuous groups, Advances in Math., 28 (1978), 186-230.

4. G. W. Mackey, Ergodic theory and virtual groups, Math. Annalen, 166 (1966), 187-207.

5. S. MacLane, Homology, Berlin, Springer, 1963.

6. C. C. Moore, Group extensions and cohomology for locally compact groups III, Trans. Amer. Math. Soc., 221 (1976), 1-33.

7. A. Ramsay, Virtual groups and group actions, Adv. Math., 6 (1971), 253-332.

8. _- Boolean duals of virtual groups, J. Functional Analysis, 15 (1974), 56-101.

9. - Subobjects of Virtual groups. Unpublished.

10. - Non-transitive quasi-orbits in Mackey's analysis of group extensions, Acta. Math., 137 (1976), 17-48.

11. K. Schmidt, Lectures on cocycles of ergodic transformation groups, 1976, Mathematics Institute, University of Warwick, Coventry.

12. J. Westman, Cohomology for the ergodic actions of countable groups, Proc. Amer. Math. Soc., 30 (1970), 318-320.

Received November 20, 1977. Supported in part by NSF grant MCS 74-19876. A02.

Mathematics InSTItUTe

WARWICK UNIVERSITY

COVENTRY CV4 7AL

ENGLAND 


\section{PACIFIC JOURNAL OF MATHEMATICS}

\section{EDITORS}

DONALD BABBITT (Managing Editor)

University of California

Los Angeles, CA 90024

HUGo RossI

University of Utah

Salt Lake City, UT 84112

C. C. MOORE and ANDREW OGG

University of California

Berkeley, CA 94720
J. DUGUNDJI

Department of Mathematics

University of Southern California

Los Angeles, CA 90007

R. FinN and J. Milgram

Stanford University

Stanford, CA 94305

\section{ASSOCIATE EDITORS}
R. ARENS
E. F. BECKENBACH
B. H. NeumanN
F. WOLF
K. Yoshida

\section{SUPPORTING INSTITUTIONS}

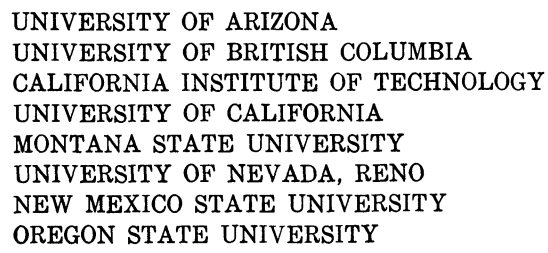

UNIVERSITY OF ARIZONA

UNIVERSITY OF BRITISH COLUMBIA CALIFORNIA INSTITUTE OF TECHNOLOGY

UNIVERSITY OF CALIFORNIA

MONTANA STATE UNIVERSITY

UNIVERSITY OF NEVADA, RENO

NEW MEXICO STATE UNIVERSITY

OREGON STATE UNIVERSITY

\author{
UNIVERSITY OF OREGON \\ UNIVERSITY OF SOUTHERN CALIFORNIA \\ STANFORD UNIVERSITY \\ UNIVERSITY OF HAWAII \\ UNIVERSITY OF TOKYO \\ UNIVERSITY OF UTAH \\ WASHINGTON STATE UNIVERSITY \\ UNIVERSITY OF WASHINGTON
}

The Supporting Institutions listed above contribute to the cost of publication of this Journal, but they are not owners or publishers and have no responsibility for its content or policies.

Mathematical papers intended for publication in the Pacific Journal of Mathematics should be in typed form or offset-reproduced, (not dittoed), double spaced with large margins. Please do not use built up fractions in the text of the manuscript. However, you may use them in the displayed equations. Underline Greek letters in red, German in green, and script in blue. The first paragraph or two must be capable of being used separately as a synopsis of the entire paper. Please propose a heading for the odd numbered pages of less than 35 characters. Manuscripts, in triplicate, may be sent to any one of the editors. Please classify according to the scheme of Math. Reviews, Index to Vol. 39. Supply name and address of author to whom proofs should be sent. All other communications should be addressed to the managing editor, or Elaine Barth, University of California, Los Angeles, California, 90024.

50 reprints to each author are provided free for each article, only if page charges have been substantially paid. Additional copies may be obtained at cost in multiples of 50 .

The Pacific Journal of Mathematics is issued monthly as of January 1966. Regular subscription rate: $\$ 102.00$ a year (6 Vols., 12 issues). Special rate: $\$ 51.00$ a year to individual members of supporting institutions.

Subscriptions, orders for numbers issued in the last three calendar years, and changes of address shoud be sent to Pacific Journal of Mathematics, P.O. Box 969, Carmel Valley, CA 93924, U.S.A Old back numbers obtainable from Kraus Periodicals Co., Route 100, Millwood, NY 10546.

\section{PUBLISHED BY PACIFIC JOURNAL OF MATHEMATICS, A NON-PROFIT CORPORATION}

Printed at Kokusai Bunken Insatsusha (International Academic Printing Co., Ltd.). 8-8, 3-chome, Takadanobaba, Shinjuku-ku, Tokyo 160, Japan. 


\section{Pacific Journal of Mathematics}

Vol. 92, No. $2 \quad$ February, 1981

Bruce Allem Anderson and Philip A. Leonard, Sequencings and Howell designs

Kevin T. Andrews, Representation of compact and weakly compact

operators on the space of Bochner integrable functions . . . . . . . . 257

James Glenn Brookshear, On the structure of hyper-real $z$-ultrafilters . . . . . 269

Frank John Forelli, Jr., A necessary condition on the extreme points of a class of holomorphic functions. II ...................... 277

Richard J. Friedlander, Basil Gordon and Peter Tannenbaum, Partitions of groups and complete mappings ......................... 283

Emden Robert Gansner, Matrix correspondences of plane partitions ......295

David Andrew Gay and William Yslas Vélez, The torsion group of a radical extension ..........................................

André (Piotrowsky) De Korvin and C. E. Roberts, Convergence theorems for some scalar valued integrals when the measure is Nemytskii ...... 329

Takaŝi Kusano and Manabu Naito, Oscillation criteria for general linear ordinary differential equations $\ldots \ldots \ldots \ldots \ldots \ldots \ldots \ldots \ldots \ldots \ldots \ldots \ldots \ldots \ldots \ldots$

Vo Thanh Liem, Homotopy dimension of some orbit spaces .......... 357

Mark Mahowald, $b o$-resolutions . . . . . . . . . . . . . . . . . . . 365

Jan van Mill and Marcel Lodewijk Johanna van de Vel, Subbases, convex

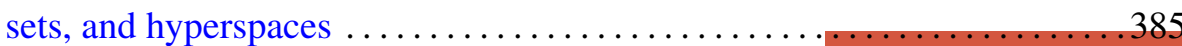

John F. Morrison, Approximations to real algebraic numbers by algebraic numbers of smaller degree $\ldots \ldots \ldots \ldots \ldots \ldots \ldots \ldots \ldots \ldots \ldots \ldots \ldots$

Caroline Series, An application of groupoid cohomology . . . . . . . . . . 415

Peter Frederick Stiller, Monodromy and invariants of elliptic surfaces . . . 433 Akihito Uchiyama, The factorization of $H^{p}$ on the space of homogeneous

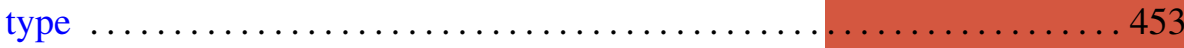

Warren James Wong, Maps on simple algebras preserving zero products.

II. Lie algebras of linear type 